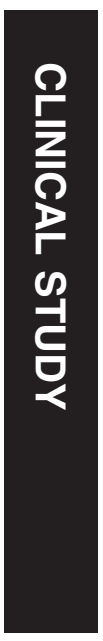

\title{
Microperimetric sensitivity in patients on hydroxychloroquine (Plaquenil) therapy
}

${ }^{1}$ Department of Ophthalmology and Visual Sciences, University of Illinois Chicago, Chicago, IL, USA

${ }^{2}$ Pangere Center for Hereditary Retinal Diseases, The Chicago Lighthouse for People Who Are Blind or Visually Impaired, Chicago, IL, USA

${ }^{3}$ Allergan Inc., Irvine, CA, USA

Correspondence: R Jivrajka, Department of Ophthalmology and Visual Sciences, University of Illinois Chicago, 1855 W Taylor Street, MC 648, Chicago, IL 60612, USA Tel: +1 312996 6562; Fax: +1 3129961934 .

E-mail: renujiv@gmail.com

This material was previously presented at the Associate for Research in Vision and Ophthalmology, Fort Lauderdale, May 2012.

${ }^{4}$ These authors contributed equally to this work

Received: 11 March 2013 Accepted: 23 April 2013 Published online: 14 June 2013

\begin{abstract}
Purpose The purpose of this study was to measure macular sensitivity using microperimetry in patients on Plaquenil therapy without evidence of retinopathy as assessed by recommended screening standards. Methods Sixteen patients from a clinical practice treated with 200 or $400 \mathrm{mg} /$ day of Plaquenil for more than 5 years, without visual complaints (visual acuity $20 / 25$ or better), and without a history of diabetes or macular disease were included. Participants underwent a complete ophthalmic examination with spectral-domain optical coherence tomography (SD-OCT), 10-2 Humphrey visual field (HVF), fundus autofluoresecene (FAF), multifocal electroretinography (mfERG), and microperimetry that covered the central $12^{\circ}$ of the visual field. Ten age-similar, visually normal subjects served as controls.

Results The average age of the study cohort was of 54.5 years, with an average daily Plaquenil dose of $4.00 \mathrm{mg} / \mathrm{kg} /$ day (range, $1.77-$ $6.67 \mathrm{mg} / \mathrm{kg} /$ day) and an average cumulative dose of $1485 \mathrm{~g}$ (range, 256-3650 g). All patients had normal ocular exams, and no evidence of retinopathy based on 10-2 HVF, FAF, mfERG, and SD-OCT. The mean retinal sensitivity by microperimetry was $15.0 \mathrm{~dB}(\mathrm{OD})$ and $14.6 \mathrm{~dB}$ (OS). The overall mean microperimetry sensitivity of the patients $(14.7 \pm 1.9 \mathrm{~dB})$ was significantly lower $(P<0.001)$ than that of the controls $(16.5 \pm 2.1 \mathrm{~dB})$.

Conclusions Patients on Plaquenil without clinical evidence of retinal toxicity can have reduced retinal sensitivity, as assessed by microperimetry. The mean sensitivity difference between the patients and controls suggests that microperimetry can provide
\end{abstract}

RV Jivrajka',4, MA Genead'2,3,4, JJ McAnany', CC Chow ${ }^{1}$ and WF Mieler ${ }^{1}$ important information regarding visual function in patients on Plaquenil therapy. Eye (2013) 27, 1044-1052; doi:10.1038/eye.2013.112; published online 14 June 2013

Keywords: plaquenil toxicity; microperimetry; retinopathy; screening

\section{Introduction}

Thousands of patients currently use hydroxychloroquine (Plaquenil; Sanofi-Aventis, Bridgewater, NJ, USA) for the management of various connective tissue disorders, such as rheumatoid arthritis (RA) and systemic lupus erythematosus (SLE). ${ }^{1}$ This medication is known to have a high affinity for binding to melanin granules and can therefore accumulate in the iris, choroid, ciliary body, and retinal pigment epithelium (RPE) on prolonged usage. ${ }^{1-3}$

Although not entirely understood, retinal toxicity often begins with loss of rods and cones and degenerative changes of the RPE. Given the concern for retinal toxicity from Plaquenil use, early detection is an important responsibility faced by ophthalmologists. The American Academy of Ophthalmology has recently published revised screening guidelines to identify tests that provide the most useful information in detecting retinal toxicity from the use of Plaquenil. ${ }^{1}$ The current standard of care based on this report involves a baseline ocular examination as well as 10-2 Humphrey visual fields (HVFs) and one of the three possible adjunct tests including spectraldomain optical coherence tomography (SD-OCT), fundus autofluoresecene (FAF), or multifocal electroretinography (mfERG). Color vision testing and the Amsler grid are no longer recommended screening procedures. As the risk of toxicity 
approaches $1 \%$ after more than 7 years of Plaquenil usage, annual screening is recommended for patients exceeding 5 years of exposure. ${ }^{4}$ Early retinopathy is associated with an acquired paracentral visual field threshold elevation without any observable fundus changes.,

Current screening methods for Plaquenil retinopathy are limited in that some detect toxicity only after significant structural damage has occurred, as seen on funduscopic examination, SD-OCT, and FAF. The need for comprehensive ophthalmic screening and early detection of retinopathy has a critical role in the surveillance of patients on chronic Plaquenil therapy. Patients often exceed the recommended daily dosage based on lean body weight, even when prescribed the usual daily dose of $400 \mathrm{mg}$ /day, leading to devastating visual consequences. ${ }^{7}$ From a functional standpoint, 10-2 HVF is a subjective test that is widely available, although test reliability is limited by fixation instability and patient cooperation. Recently, mfERG has been shown to detect subtle changes in earlier stages of toxicity. ${ }^{8}$ However, mfERG is limited by clinical availability, patient cooperation, specialized training for administration and interpretation, and cost. A non-invasive, sensitive, and reproducible test such as microperimetry could be another option for assessing retinal sensitivity in Plaquenil users. Although there exists no single definitive test, it is important to investigate detailed and comprehensive screening methods with the goal of detecting retinopathy, defined as structural or functional changes, before irreversible vision loss occurs.

The introduction of microperimetry testing with a scanning laser ophthalmoscope (SLO) using the OPKO microperimeter (OPKO Instrumentations, Miami, FL, USA) provides clinicians with an additional tool to evaluate macular function in conjunction with SD-OCT. The microperimeter incorporates automated ocular tracking capabilities, which compensate for eye movements under real-time conditions and permits good test reliability and reproducibility. ${ }^{9}$ The operating system directly relates perimetric sensitivity values to SLO infrared images and associated SD-OCT thickness values by superimposing the acquired data, thus allowing direct correlations of structural and functional abnormalities. The purpose of this study is to examine retinal sensitivity and thickness in patients who are on Plaquenil for more than 5 years with no evidence of retinopathy based on 10-2 HVF, SD-OCT, FAF, and mfERG testing.

\section{Patients and methods}

\section{Patient selection}

All patients were seen at a clinical practice and had been taking Plaquenil (100-400 mg/day) for a minimum of 5 years (range, 5-25 years) before presentation. These patients had no visual complaints and no clinical evidence of retinopathy. The study and data collection were carried out with prospective approval from an Institutional Review Board at the University of Illinois Chicago, informed consent for the research was obtained from the subjects, and the study is in accordance with HIPAA regulations. Medical history, including reason for Plaquenil usage, was recorded from the patient's medical record. Ocular exam details including best-corrected visual acuity (BCVA), intraocular pressure measurements using Goldmann applanation tonometry, color vision testing using Ishihara pseudoisochromatic color plates, slit-lamp biomicroscopy, and dilated fundus examination were recorded. Patients also underwent testing using the Humphrey 10-2 visual field, Heidelberg Spectralis SD-OCT, and Heidelberg Spectralis FAF, and mfERG (VERIS system). Patients with visual complaints or BCVA worse than 20/25, history of diabetes or hypertension, weight fluctuation $>20 \%$ within 1 year, or any macular diseases, including Plaquenil retinopathy, were excluded from the study. Patients who met the inclusion criteria and had normal results from the above tests underwent microperimetry and SD-OCT testing with the OPKO instrument.

\section{Heidelberg SD-OCT and FAF testing}

Both SD-OCT and FAF were performed by the same skilled technician using a commercially available instrument (Spectralis; Heidelberg Engineering, Heidelberg, Germany). High-resolution OCT images were obtained using a pattern size of $20^{\circ}(5.9 \mathrm{~mm})$. A total of 19 B-scans were obtained from each eye. Realtime FAF images were recorded over a $30^{\circ}$ field of view. An excitatory argon laser beam at $488 \mathrm{~nm}$ and a barrier filter at $500 \mathrm{~nm}$ were coupled in a collimating system for detecting FAF emission at wavelengths above $500 \mathrm{~nm}$. To more optimally visualize the distribution of FAF intensities over the fundus, the pixel value distribution was normalized after calculation of the mean image. All patients underwent initial screening using the Spectralis SD-OCT unit before referral on a subsequent visit for MP testing and repeat SD-OCT testing using the OPKO Spectral OCT/SLO system.

\section{Microperimetry testing}

Microperimetry was performed using the OPKO Spectral OCT/SLO system by the same examiner (MAG). All patients underwent testing in both eyes, with the exception of one patient who declined testing in the second eye. For those patients with reduced retinal sensitivities on initial testing, reproducibility was 
Table 1 Summary of clinical data

\begin{tabular}{|c|c|c|c|c|c|c|c|}
\hline Subject no. & Age (years) & Race & Sex & $\begin{array}{l}\text { Systemic } \\
\text { diagnosis }\end{array}$ & $\begin{array}{l}\text { Years of } \\
\text { exposure }\end{array}$ & $\begin{array}{l}\text { Maximum daily } \\
\text { dose }(m g / k g / \text { day })\end{array}$ & $\begin{array}{c}\text { Cumulative } \\
\text { dose }(\mathrm{g})\end{array}$ \\
\hline 1 & 76 & AA & F & RA & 7 & 1.76 & 256 \\
\hline 2 & 44 & $\mathrm{H}$ & $\mathrm{F}$ & SLE/Sjogrens & 5 & 2.59 & 365 \\
\hline 3 & 63 & Asian & $\mathrm{F}$ & RA & 5 & 1.76 & 365 \\
\hline 4 & 54 & AA & $\mathrm{F}$ & RA & 7 & 1.38 & 511 \\
\hline 5 & 43 & AA & $\mathrm{F}$ & SLE & 5 & 4.41 & 730 \\
\hline 6 & 62 & $\mathrm{H}$ & $\mathrm{M}$ & SLE & 11 & 3.39 & 803 \\
\hline 7 & 60 & $\mathrm{H}$ & $\mathrm{F}$ & Dermatomyositis & 6 & 4.63 & 876 \\
\hline 8 & 37 & AA & F & SLE & 8 & 3.88 & 1168 \\
\hline 9 & 61 & AA & F & SLE & 11 & 6.23 & 1606 \\
\hline 10 & 57 & AA & $\mathrm{F}$ & SLE & 12 & 3.21 & 1606 \\
\hline 11 & 52 & AA & $\mathrm{F}$ & SLE & 12 & 5.68 & 1752 \\
\hline 12 & 37 & AA & $\mathrm{F}$ & SLE & 13 & 5.4 & 1898 \\
\hline 13 & 59 & AA & $\mathrm{F}$ & RA & 17 & 6.77 & 2482 \\
\hline 14 & 51 & AA & F & SLE & 19 & 4.96 & 2774 \\
\hline 15 & 60 & AA & F & SLE & 20 & 3.53 & 2920 \\
\hline 16 & 56 & AA & F & SLE & 25 & 4.37 & 3650 \\
\hline
\end{tabular}

Abbreviations: AA, African American; H, Hispanic; RA, rheumatoid arthritis; SLE, systemic lupus erythematosus.

confirmed by a second test on each eye. Following dilation with $2.5 \%$ phenylephrine and $1 \%$ tropicamide, the subjects were seated in a darkened room and instructed to fixate on a red square with the tested eye while the non-tested eye was patched. Patients were then asked to press a button each time they saw the stimulus, which consisted of a spot of light that was equivalent in size to the Goldmann III (area $4 \mathrm{~mm}^{2}$, diameter $26 \mathrm{~min}$ of arc, or $0.4^{\circ}$ ). The stimulus was presented for $200 \mathrm{~ms}$ using a 4-2 test strategy to estimate threshold. The Polar 3 testing grid was used in all patients, which is a standardized grid of 28 points forming three concentric circles $\left(2.3^{\circ}, 6.6^{\circ}\right.$, and $\left.11^{\circ}\right)$. The inner circle is made of 4 points, while the middle and outer circles are composed of 12 points each. Sensitivity values were measured in 10 age-similar, visually normal subjects with the same instrumentation for comparison with our patient cohort.

\section{SD-OCT retinal thickness mapping}

SD-OCT was performed using the spectral OCT/SLO system (OPKO Instrumentations) to obtain both OCT and SLO images with an axial resolution of $<10 \mu \mathrm{m}$, and a transverse resolution of $20 \mu \mathrm{m}$ (in tissue). The system uses an SLO fundus image for alignment, orientation, and registration of the OCT image topographic maps. Both the Line scan (B-scan) and the 3-Dimensional Retinal Topography scan protocols were used for image acquisition. The Line scan mode allows the capture of cross-sectional B-scan OCT images of the vitreoretinal, retinal, and chorioretinal structures. A red scanning line on the SLO image indicated the exact location of the cross-sectional OCT image. We used the 'Max Frame Count' of 32 frames. The 'Max Frame Count' is the maximum sequentially captured frames of OCT and SLO images, which are captured and displayed as individual frames. The 3D Retinal Topography mode covers an area of $9.0 \times 9.0 \mathrm{~mm}^{2}$ with a $2.0 \mathrm{~mm}$ depth. After retinal thickness and microperimetry maps were generated, the images were aligned using native software so that the retinal thickness at each MP test location is displayed. The OCT data acquired by the OPKO system were used for analysis in the present study.

\section{Multifocal ERG testing}

MfERG was performed on both eyes in 14 of 16 subjects using the International Society for Clinical Electrophysiology of Vision guidelines. ${ }^{10}$ Two patients were lost to follow-up before completing mfERG testing. Following dilation using 2.5\% phenylephrine and $1 \%$ tropicamide, recordings were performed unilaterally using a unipolar Burian-Allen contact lens electrode. MfERGs were recorded and analyzed using the VERIS Clinic system (Electro-Diagnostic Imaging, Inc., Redwood, CA, USA). Testing was completed in a darkened room by the same trained technician for all subjects. The stimulus consisted of a black-and-white pattern of 61 hexagons that was presented on a monitor $\left(200 \mathrm{~cd} / \mathrm{m}^{2}\right.$ for white, $99.3 \%$ contrast). The duration of data acquisition involved eight short intervals lasting a total of $4-5 \mathrm{~min}$.

\section{Data analysis}

A three-way analysis of variance (ANOVA), with Bonferroni-corrected follow-up comparisons, was performed to examine differences in retinal sensitivity, as assessed by microperimetry, between the patient and control groups 
Table 2 Summary of testing results

\begin{tabular}{|c|c|c|c|c|c|c|c|c|c|}
\hline $\begin{array}{l}\text { Subject } \\
\text { no. }\end{array}$ & Eye Lc & ogMAR & Fundus & $\begin{array}{c}\text { Ishihara color } \\
\text { plates }\end{array}$ & $\begin{array}{l}\text { HVF } \\
10-2\end{array}$ & $F A F$ & $m f E R G$ & $\begin{array}{l}\text { Retinal thickness } \\
(S D-O C T)\end{array}$ & $\begin{array}{l}M P \\
(d B)\end{array}$ \\
\hline 1 & OS & 0.02 & $\begin{array}{l}\text { Punctate } \\
\text { drusen }\end{array}$ & $17 / 17$ & Full & Normal & wnl & Normal & 12.4 \\
\hline 2 & OD & 0 & wnl & $17 / 17$ & Full & Normal & wnl & Normal & 16.2 \\
\hline 2 & OS & 0 & wnl & $17 / 17$ & Full & Normal & $\begin{array}{c}\text { Not } \\
\text { completed }\end{array}$ & Normal & 16 \\
\hline 3 & OD & 0 & wnl & $17 / 17$ & Full & Normal & $\begin{array}{c}\text { Not } \\
\text { completed }\end{array}$ & Normal & 13.7 \\
\hline 3 & OS & 0 & wnl & $17 / 17$ & Full & Normal & $\begin{array}{c}\text { Not } \\
\text { completed }\end{array}$ & Normal & 12.6 \\
\hline 4 & OD & 0.02 & wnl & $17 / 17$ & Full & Normal & $\begin{array}{c}\text { Not } \\
\text { completed }\end{array}$ & Normal & 16.9 \\
\hline 4 & OS & 0 & $\begin{array}{l}\text { Punctate } \\
\text { drusen }\end{array}$ & $17 / 17$ & Full & Normal & $\begin{array}{c}\text { Not } \\
\text { completed }\end{array}$ & Normal & 15.5 \\
\hline 5 & OD & 0.06 & wnl & $17 / 17$ & Full & Normal & wnl & Normal & 14.9 \\
\hline 5 & OS & 0 & wnl & $17 / 17$ & Full & Normal & wnl & Normal & 16.2 \\
\hline 6 & OD & 0.02 & $\begin{array}{l}\text { Punctate } \\
\text { drusen }\end{array}$ & $17 / 17$ & Full & Normal & wnl & Normal & 15.5 \\
\hline 6 & OS & 0 & $\begin{array}{l}\text { Punctate } \\
\text { drusen }\end{array}$ & $17 / 17$ & Full & $\begin{array}{l}\text { Temporal hypofluorescence } \\
\text { corresponding to drusen }\end{array}$ & wnl & Normal & 16.2 \\
\hline 7 & OD & 0.02 & wnl & $17 / 17$ & Full & Normal & wnl & Normal & 13.4 \\
\hline 7 & OS & 0.02 & wnl & $17 / 17$ & Full & Normal & wnl & Normal & 14.4 \\
\hline 8 & OD & 0 & wnl & $17 / 17$ & Full & Normal & wnl & Normal & 16.1 \\
\hline 8 & OS & 0 & wnl & $17 / 17$ & Full & Normal & wnl & Normal & 16.1 \\
\hline 9 & OD & 0.02 & wnl & $17 / 17$ & Full & Normal & wnl & Normal & 14.3 \\
\hline 9 & OS & 0.15 & $\begin{array}{l}\text { Extramacular } \\
\text { drusen }\end{array}$ & $16 / 17$ & Full & Normal & wnl & Normal & 12.8 \\
\hline 10 & OD & -0.05 & $\begin{array}{l}\text { Punctate } \\
\text { drusen }\end{array}$ & $17 / 17$ & Full & Normal & wnl & Normal & 14.9 \\
\hline 10 & OS & -0.07 & $\begin{array}{l}\text { Punctate } \\
\text { drusen }\end{array}$ & $17 / 17$ & Full & Normal & wnl & Normal & 14.7 \\
\hline 11 & OD & 0 & wnl & $17 / 17$ & Full & $\begin{array}{l}\text { Inferior hypofluorescence } \\
\text { corresponding to drusen }\end{array}$ & wnl & $\begin{array}{c}\text { Normal, extrafoveal } \\
\text { drusen }\end{array}$ & 14.1 \\
\hline 11 & OS & 0 & $\begin{array}{l}\text { Punctate } \\
\text { drusen }\end{array}$ & $17 / 17$ & Full & $\begin{array}{l}\text { Inferior hypofluorescence } \\
\text { corresponding to drusen }\end{array}$ & wnl & $\begin{array}{l}\text { Normal, extrafoveal } \\
\text { drusen }\end{array}$ & 15.1 \\
\hline 12 & OD & 0 & wnl & $17 / 17$ & Full & Normal & wnl & Normal & 14.1 \\
\hline 12 & OS & 0 & wnl & $17 / 17$ & Full & Normal & wnl & Normal & 14.5 \\
\hline 13 & OD & 0 & wnl & $17 / 17$ & Full & Normal & wnl & Normal & 15.1 \\
\hline 13 & OS & 0.02 & wnl & $17 / 17$ & Full & Normal & wnl & Normal & 14.1 \\
\hline 14 & OD & 0 & wnl & $17 / 17$ & Full & Normal & wnl & Normal & 15.1 \\
\hline 14 & OS & 0 & wnl & $17 / 17$ & Full & Normal & wnl & Normal & 13.6 \\
\hline 15 & OD & 0.1 & wnl & $17 / 17$ & Full & Normal & wnl & Normal & 13.5 \\
\hline 15 & OS & 0 & wnl & $17 / 17$ & Full & Normal & wnl & Normal & 14.4 \\
\hline 16 & OD & 0 & wnl & $17 / 17$ & Full & Normal & wnl & Normal & 16.7 \\
\hline 16 & OS & 0 & wnl & $17 / 17$ & Full & Normal & wnl & Normal & 16 \\
\hline
\end{tabular}

Abbreviations: BCVA, best-corrected visual acuity; dB, decibels; FAF, fundus autofluorescence; HVF, Humphrey visual fields; mfERG, multifocal electroretinogram; MP, microperimetry; SD-OCT, spectral-domain optical coherence tomography.

(with a $P$-value $<0.05$ considered significant). Subject group (patient vs control), stimulus eccentricity (inner, middle, and outer ring), and stimulus location (1 o'clock through 12 o'clock) were included as main factors.

\section{Results}

\section{Demographic characteristics}

The mean age of the patients was $54.5 \pm 10.3$ years (range, 37-76 years) with 15 female subjects and 1 male subject. Twelve $(75 \%)$ of the subjects were African American, 3 (19\%) were Hispanic, and $1(6 \%)$ was Asian. Of the 16 patients with a history of Plaquenil usage, 11 had a diagnosis of SLE, 4 with RA, and 1 with dermatomyositis (Table 1). The mean age of the visually normal control subjects was $53.4 \pm 11.9$ years (range, 31-65 years). The mean age of the patients did not differ significantly from that of the controls $(P=0.81)$.

The duration of Plaquenil exposure ranged from 5-25 years (median, 11 years). The average maximum daily dose for the study population was $4 \mathrm{mg} / \mathrm{kg}$ /day (range, $1.77-6.67 \mathrm{mg} / \mathrm{kg} /$ day) with an average cumulative dose of $1485 \mathrm{~g}$ (range, 255-3650 g). The BCVA ranged from -0.07 to $0.15 \log$ MAR (Snellen acuity equivalent of $20 / 20^{+3}$ to $20 / 25^{-3}$ ). The funduscopic exam was normal in all subjects, as were the results from color vision testing. The 10-2 HVF results were graded by three authors independently (MAG, CCC, and RVJ). There was no evidence of retinopathy based on the pattern deviation maps, but, overall sensitivity was reduced slightly for the patients based on the mean deviation value. One patient had scattered outer rim defects on her visual field that were considered to be within the normal range. Both FAF 


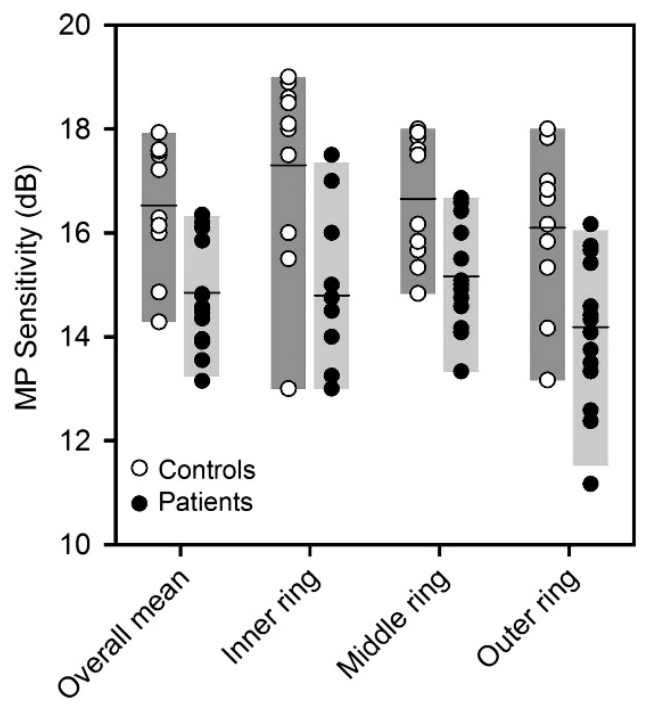

Figure 1 Mean sensitivity for the control subjects (open circles) and patients (filled circles) for the overall mean and each of the three rings. The bars represent the 5th and 95th percentiles, and the horizontal bars represent the mean for each group.

and SD-OCT exams, graded by the retina specialists independently (MAG, CCC, and WFM), showed normal autofluorescence and retinal structures and thickness, respectively. Two patients were noted to have few extrafoveal drusen outside the Polar 3 test grid, on FAF and SD-OCT that correlated with their funduscopic exams. Of the 14 patients who underwent mfERG testing, a qualitative analysis of the results indicated that all had a normal waveform shape (Table 2).

\section{Microperimetry data}

The overall mean sensitivity values for the right eye $(15.0 \pm 1.1 \mathrm{~dB})$ and left eye $(14.6 \pm 1.2 \mathrm{~dB})$ of the 15 patients who had measurements in both eyes were not significantly different $(t=0.87, P=0.40)$. Consequently, data from the left eye were converted to right eye format and the data from the two eyes of each patient were averaged. All further analysis was based on the mean sensitivity value of the two eyes. The microperimetry sensitivity data are shown in Figure 1 for the controls (open circles) and the patients (filled circles). The bars represent the 5th and 95th percentiles for the controls (dark bars) and the patients (light bars), and the horizontal lines indicate the mean for each group. A three-way ANOVA indicated a significant difference $(P<0.001)$ only for the main effect of subject group (overall mean sensitivities of $16.5 \pm 2.1$ and $14.7 \pm 1.9 \mathrm{~dB}$ for the controls and patients, respectively). The overall microperimetry value was below the 5 th percentile of the controls for 5 of the 16 patients (first set of bars in Figure 1). For the inner ring (second set of bars), the microperimetry value for each patient fell within the 5th and 95th percentiles of the controls. For the middle ring (third set of bars), six patients were below the 5th percentile of the controls, and three patients were below the 5th percentile for the outer ring (fourth set of bars). Bonferroni-corrected follow-up comparisons indicated that the mean sensitivity values within the inner (Figure 1: second pair of bars), middle (Figure 1: third pair of bars), and outer (Figure 1: fourth pair of bars) rings were significantly less for the patients, as compared with the controls $(P=0.001, P=0.004, P=0.003$, for the inner, middle, and outer rings, respectively). The sensitivity values for the patients did not significantly differ at the three eccentricities (mean sensitivities of $15.8 \pm 2.3,15.7 \pm 1.9$, and $14.9 \pm 2.3 \mathrm{~dB}$ for the inner, middle, and outer rings, respectively) or among the retinal locations within a ring.

\section{SD-OCT data/thickness map}

The overall mean thickness for right eye $(281.0 \pm 14.2 \mu \mathrm{m})$ and left eye $(279.6 \pm 24.3 \mu \mathrm{m})$ of the 15 patients who had measurements in both eyes was not significantly different $(t=0.34, P=0.74)$. Consequently, data from the left eye were converted to right eye format and the data from the two eyes of each patient were averaged. The overall mean thickness of the controls $(287.2 \pm 14.0 \mu \mathrm{m})$ was not significantly different from that of the patients $(280.3 \pm 18.3 \mu \mathrm{m})(P=0.32)$. Bonferroni-corrected followup comparisons indicated that the mean thickness values within the inner, middle, and outer rings were not significantly different for the patients, as compared with the controls ( $P=0.17, P=0.07, P=0.99$, for the inner, middle, and outer rings, respectively).

Figures 2 and 3 summarize the OCT, microperimetry, HVF pattern deviation, and FAF results for two representative patients.

\section{Discussion}

The current standard of care based on the recommended screening guidelines for patients on Plaquenil therapy requires the use of one of three objective screening tests including SD-OCT, FAF, or mfERG in addition to 10-2 HVF. Careful monitoring of these patients is needed, as long-term use of Plaquenil can result in bull's eye maculopathy and loss of retinal function. Although patients with normal findings under the recommended screening guidelines are considered to have normal visual function, the data from the current study suggest that these patients can, as a group, have small but statistically significant reductions in microperimetry sensitivity. All patients in the present study had 10-2 HVF pattern deviations that had no clinically significant defects, minimal risk factors for toxicity including renal 


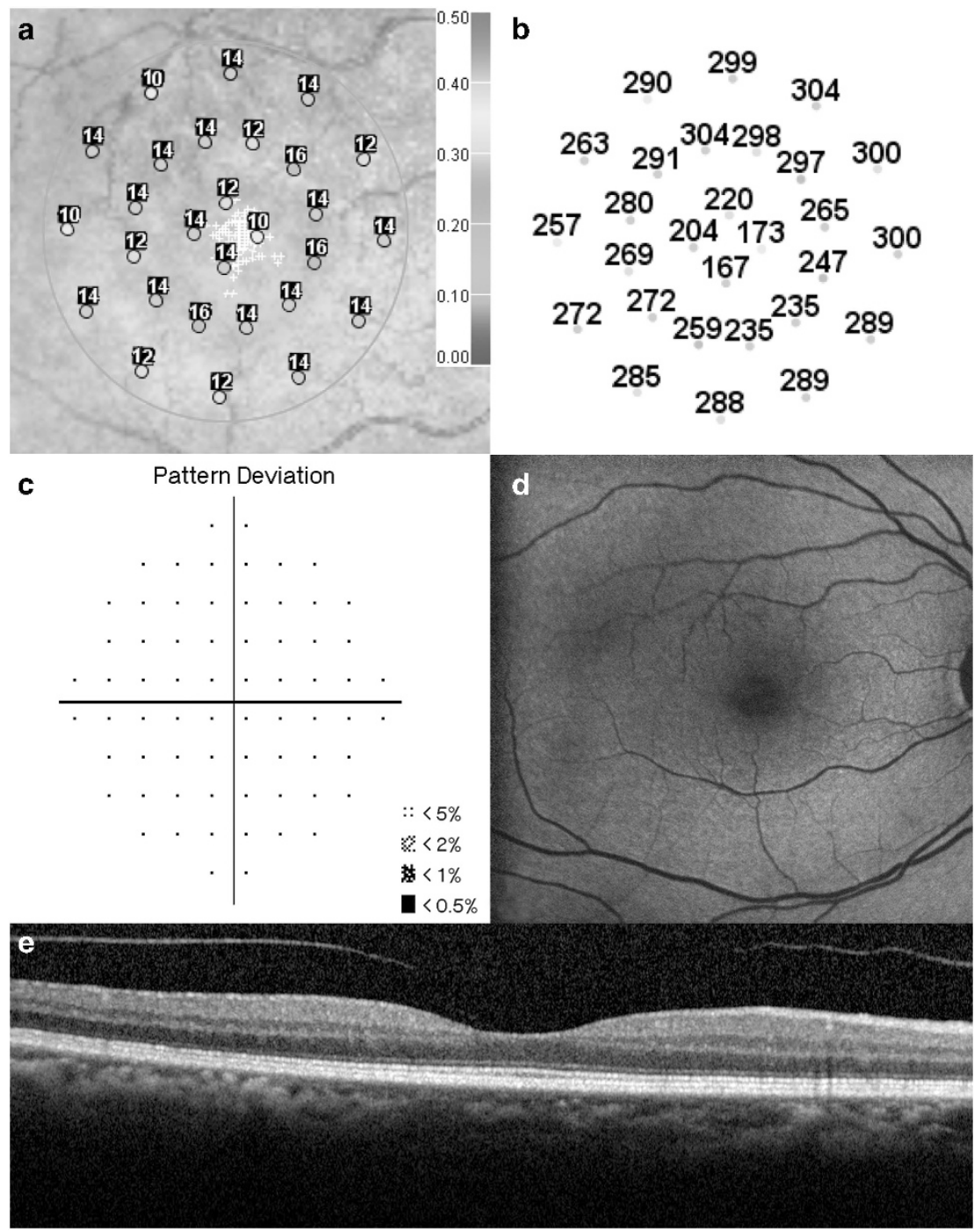

Figure 2 Test results of a 60-year-old Hispanic female on Plaquenil for 6 years for treatment of dermatomyositis (maximum daily dose of $4.63 \mathrm{mg} / \mathrm{kg} /$ day and cumulative dose of $876 \mathrm{~g}$, BCVA 20/20-1). (a) Microperimetry Polar 3 test grid superimposed on the scanning laser ophthalmoscope infrared image showing reduced mean retinal sensitivity $(13.4 \mathrm{~dB})$. Small white crosses indicate fixation. (b) Corresponding spectral-domain optical coherence tomography thickness at each of the 28 Polar 3 tested points shows no retinal thinning. (c)Automated 10-2 Humphrey visual field shows a normal pattern deviation. (d) Fundus autofluorescence shows no retinal pigment epithelium abnormalities. (e) Spectral-domain optical coherence tomography B-scan image shows normal retinal thickness and an intact inner segment-outer segment junction of the photoreceptors.

disease, liver disease, advanced age, and pre-existing retinal or macular disease, yet we found a statistically significant overall reduction in mean retinal sensitivity on microperimetry $(16.5 \pm 2.1$ and $14.7 \pm 1.9 \mathrm{~dB}$ for the controls and patients, respectively). In addition, this cohort of patients demonstrated normal outcomes on objective tests (SD-OCT, FAF, and mfERG) routinely used when screening for Plaquenil toxicity.

Plaquenil toxicity is typically evaluated by the 10-2 HVF pattern deviation, which can identify subtle cases. ${ }^{11}$ However, the mean deviation value, as an average of the reduction from normal, may also provide useful information regarding overall retinal sensitivity in these patients. Comparisons of 10-2 HVF and microperimetry have received minimal attention in the literature and a direct comparison between these testing methodologies is beyond the scope of this study. However, a previous study of glaucoma patients using microperimetry and 10-2 HVF found that the results of these techniques correlate well and that in some cases, microperimetry was able to detect abnormal areas in retinal sensitivity that 10-2 HVF did not. ${ }^{12}$ Based on this result, it was proposed that in areas of macular thinning, microperimetry might be more sensitive than standard automated perimetry in 


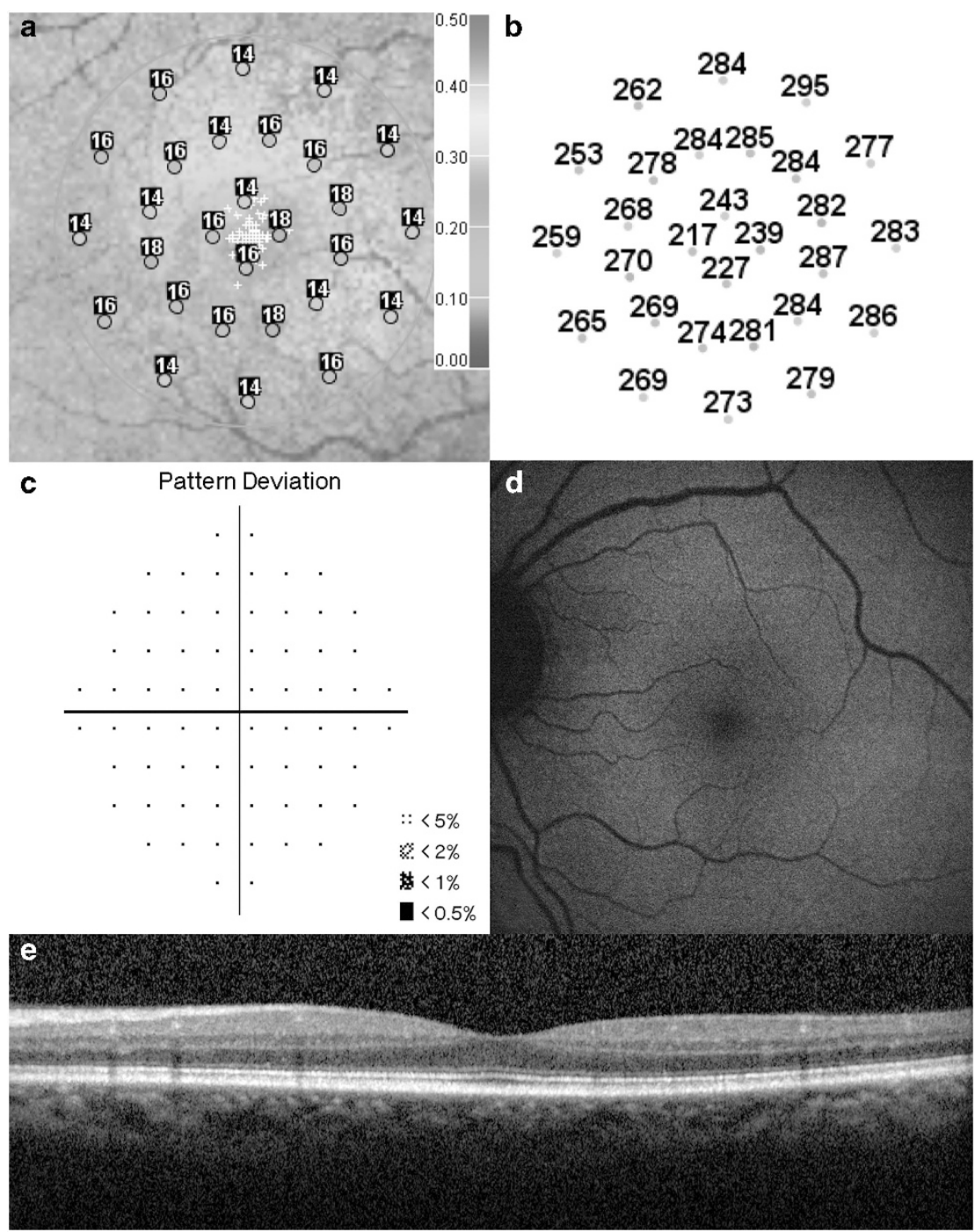

Figure 3 Test results of a 59-year-old African American female with a 17-year history of Plaquenil use for treatment of rheumatoid arthritis (maximum daily dose of $6.77 \mathrm{mg} / \mathrm{kg} /$ day and cumulative dose of $2482 \mathrm{~g}$, BCVA 20/20-1). (a) Microperimetry Polar 3 test grid superimposed on the scanning laser ophthalmoscope infrared image showing reduced mean retinal sensitivity (14.1 dB). (b) Corresponding spectral-domain optical coherence tomography thickness at each of the 28 Polar 3 tested points shows no retinal thinning. (c) Automated 10-2 Humphrey visual field shows a normal pattern deviation. (d) Fundus autofluorescence shows no retinal pigment epithelium abnormalities. (e) Spectral-domain optical coherence tomography B-scan image shows normal retinal thickness and an intact inner segment-outer segment junction of the photoreceptors.

detecting paracentral visual field loss in glaucoma patients.

An additional advantage of the microperimetry system used in the present study is its ability to obtain SD-OCT images and superimpose retinal sensitivity values on an SLO image with SD-OCT thicknesses, thus offering the ability to correlate functional responses with anatomical structure. An early sign of toxicity on SDOCT is parafoveal thinning, which may involve damage to inner retinal layers, but with the evolution of toxicity, there is a progressive loss of parafoveal photoreceptors long before any RPE damage is visible clinically. ${ }^{13}$ With advanced retinopathy, anatomical changes including perifoveal loss of the inner segment-outer segment junction of the photoreceptors, outer retinal thinning, and posterior displacement of the inner retina toward the RPE have been reported, which are indicative of a severe level of toxicity. ${ }^{14,15}$ Similar patterns of Plaquenil toxicity have been described using ultra-high-resolution OCT. ${ }^{16}$ These SD-OCT features, however, are seen largely in patients with paracentral scotomas or advanced maculopathy on HVF. None of our patients had these abnormalities. In addition, the overall mean retinal thickness of the patients in the present study did 
not differ significantly from that of the controls $(287.2 \pm 14.0$ and $280.3 \pm 18.3 \mu \mathrm{m}$ for the controls and patients, respectively).

Several reports suggest that mfERG may detect decreased retinal function, in particular, in the susceptible perifoveal retina. ${ }^{17-19}$ As a measure of retinal function, mfERG generates a topograghical map of electrophysiological activity that may help pinpoint selective areas of toxicity preferential to damage by Plaquenil consumption. The most specific waveform pattern seen in patients with Plaquenil toxicity is that of paracentral amplitude loss; however, patients have been known to show reduction in the central area alone, the peripheral area alone, and a generalized reduction. ${ }^{18,19}$ On the other hand, qualitative analysis of waveforms in the present series of patients demonstrated no identifiable pattern of waveform disruption attributable to Plaquenil toxicity. Although one limitation to the study is the lack of quantitative mfERG data comparing ring ratio data of hydroxychloroquine patients to normal control subjects, the ring ratios were compared with published normative data in which there was no statistical difference. ${ }^{20}$

While it is difficult to prevent retinal damage due to Plaquenil use, the goal of screening is to detect toxicity before any functional vision loss. The substantial variability of HVF sensitivity among Plaquenil patients who have a wide range of drug dosage and duration suggests the need for multiple screening modalities. A recent comparative study by Marmor ${ }^{21}$ summarized the results of individual screening tests in detection of early retinopathy and demonstrated that different individuals are more or less sensitive to different tests. This study recommended using multiple testing modalities in certain patients for more definitive results when there is a concern for toxicity.

In conclusion, the devastating visual consequences of Plaquenil-related retinal toxicity emphasize the importance of detailed screening. Despite our relatively small sample size, the results of this study suggest that, on average, patients on prolonged Plaquenil therapy ( $>5$ years) with normal results on recommended screening procedures can have sensitivity losses throughout the central $12^{\circ}$ of the visual field. Large-scale studies are needed to identify the factors underlying the deficits in patients with sensitivity losses, determine how common the losses are, and investigate the utility of microperimetry as a tool for screening for Plaquenil toxicity. Future longitudinal studies are needed, with repeat microperimetry testing over time, to determine if the reduced microperimetry sensitivities represent early detection of Plaquenil toxicity and whether the course of treatment should be altered in patients with microperimetry sensitivity reductions.

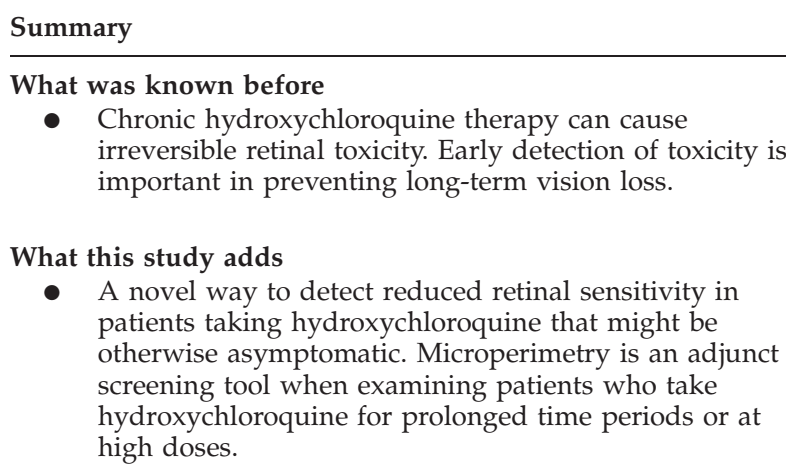

\section{Conflict of interest}

The authors declare no conflict of interest.

\section{Acknowledgements}

Fundings were obtained from the Foundation Fighting Blindness, Columbia, MD; Pangere Corporation, Gary, IN; Grant Healthcare Foundation, Lake Forest, IL; NIH core grant EY01792; NIH research grant R00EY019510 (JJM), Illinois Society for Prevention of Blindness. The sponsor or funding organization had no role in the design or conduct of this research.

\section{References}

1 Marmor MF, Kellner U, Lai TY, Lyons JS, Mieler WF. American Academy of Ophthalmology. Revised recommendations on screening for chloroquine and hydroxychloroquine retinopathy. Ophthalmology 2011; 118: $415-422$.

2 Marmor MF, Carr RE, Easterbrook M, Farjo AA, Mieler WF. American Academy of Ophthalmology. Recommendations on screening for chloroquine and hydroxychlorquine retinopathy: a report by the American Academy of Ophthalmology. Ophthalmology 2002; 109: 1377-1382.

3 Nebbioso M, Grenga R, Karavitis P.. Early detection of macular changes with multifocal ERG in patients on antimalarial drug therapy. J Ocul Pharmacol Ther 2009; 25: 249-258.

4 Mavrikakis I, Sfikakis PP, Mavrikakis E, Rougas K, Nikolaou $\mathrm{A}$, Kostopoulos $\mathrm{C}$ et al. The incidence of irreversible retinal toxicity in patients treated with hydroxychloroquine: a reappraisal. Ophthalmology 2003; 110: 1321-1326.

5 Browning DJ. Hydroxychloroquine and chloroquine retinopathy: screening for drug toxicity. Am J Ophthalmol 2002; 133: 649-656.

6 Easterbrook M. Detection and prevention of maculopathy associated with antimalarial agents. Int Ophthalmol Clin 1999; 39: 49-57.

7 Payne JF, Hubbard 3rd GB, Aaberg Sr, TM, Yan J. Clinical characteristics of hydroxychloroquine retinopathy. $\mathrm{Br} J$ Ophthalmol 2011; 95: 245-250.

8 Lyons JS, Severns ML. Detection of early hydroxychloroquine retinal toxicity enhanced by ring ratio 
analysis of multifocal electroretinography. Am J Ophthalmol 2007; 143: 801-809.

9 Menke MN, Sato E, Van De Velde FJ, Feke GT. Combined use of SLO microperimetry and OCT for retinal functional and structural testing. Graefes Arch Clin Exp Ophthalmol 2006; 244: 634-638.

10 Marmor MF, Hood DC, Keating D, Kondo M, Seeliger MW, Miyake Y. International Society for Clinical

Electrophysiology of Vision. Guidelines for basic multifocal electroretinography (mfERG). Doc Ophthalmol 2003; 106 105-115.

11 Anderson C, Blaha GR, Marx JL. Humphrey visual field findings in hydroxychloroquine toxicity. Eye (Lond) 2011; 25: 1535-1545.

12 Lima VC, Prata TS, De Moraes CG, Kim J, Seiple W, Rosen RB et al. A comparison between microperimetry and standard achromatic perimetry of the central visual field in eyes with glaucomatous paracentral visual-field defects. Br J Ophthalmol 2010; 94: 64-67.

13 Pasadhika S, Fishman GA. Effects of chronic exposure to hydroxychloroquine or chloroquine on inner retinal structures. Eye (Lond) 2010; 24: 340-346.

14 Chen E, Brown DM, Benz MS, Fish RH, Wong TP, Kim RY et al. Spectral domain optical coherence tomography as an effective screening test for hydroxychloroquine retinopathy (the 'flying saucer' sign). Clin Ophthalmol 2010; 4: 1151-1158.
15 Stepien KE, Han DP, Schell J, Godara P, Rha J, Carroll J. Spectral-domain optical coherence tomography and adaptive optics may detect hydroxychloroquine retinal toxicity before symptomatic vision loss. Trans Am Ophthalmol Soc 2009; 107: 28-32.

16 Rodriguez-Padilla JA, Hedges 3rd, TR, Monson B, Srinivasan V, Wojtkowski M, Reichel E et al. High-speed ultra-high-resolution optical coherence tomography findings in hydroxychloroquine retinopathy. Arch Ophthalmol 2007; 125: 775-780.

17 So S, Hedges T, Schuman J, Amaro Quireza ML. Evaluation of hydroxychloroquine retinopathy with multifocal electroretinography. Ophthalmic Surg Lasers Imaging 2003; 34: 251-258.

18 Maturi R, Yu M, Weleber R. Multifocal electroretinographic evaluation of long-term hydroxychloroquine users. Arch Ophthalmol 2004; 122: 973-981.

19 Lai T, Chan W, Li H, Lai RY, Lam DS. Multifocal electroretinographic changes in patients receiving hydroxychloroquine therapy. Am J Ophthalmol 2005; 140: 794-807.

20 Lyons J, Severns M. Using multifocal ERG ring ratios to detect and follow Plaquenil retinal toxicity: a review. Doc Ophthalmol 2009; 118: 29-36.

21 Marmor MF. Comparison of screening procedures in hydroxychloroquine toxicity. Arch Opthalmol 2012; 130: 461-469. 